\title{
A Yield Model of Polymers Based on the Multiplication of Deformed Region
}

\author{
Zongyan $\mathrm{HE}^{\dagger}$ and Kehchi HwaNG \\ Department of Engineering Mechanics, Tsinghua University, \\ Beijing 100084, People's Republic of China
}

(Received September 26, 1994)

\begin{abstract}
In this paper the authors proposed a new yield model of polymers based on the multiplication of deformed region, from which an explicit elastic-plastic constitutive equation can be deduced. The characteristics of stress-strain curve in different conditions are discussed and several typical yield modes can be described, which are better consistent with the experimental results. KEY WORDS Polymers / Yield / Constitutive Relation / Multiplication /
\end{abstract}

Researchers have proposed many theories to explain the yield phenomena of polymers. Considere suggested that the yield is a geometrical unstable effect based on the neckingdown of the specimen. This theory can not explain the yield without necking-down under pressure. ${ }^{1-3}$ Many people think the intristic mechanism of yield is the local increase of temperature in the deformation process. The platform of yield shows an equilibrium between the created heat and the transmitted heat in the region of the specimen. ${ }^{4-6}$ But the temperature may not be the most essential reason, because a very slow loading under constant temperature can also cause an obvious upper yield point. ${ }^{7}$

One of the micro-yield theories suggests that the external load causes molecules to flow along a direction, the yield stress is the critical one at which the strain rate is equal to the plastic strain rate $\dot{\varepsilon}_{\mathrm{p}}$ governed by the Erying's equation. On the basic of this theory, many models were developed and the following formula can be deduced ${ }^{8}$ :

$$
\frac{\sigma_{\mathrm{s}}}{T}=\frac{R}{V}\left[\frac{\Delta H}{R T}+\ln \frac{\dot{\varepsilon}_{\mathrm{s}}}{\dot{\varepsilon}_{0}}\right]
$$

where, $\sigma_{\mathrm{s}}$ is the yield stress, $\dot{\varepsilon}_{\mathrm{s}}$ is the corresponding strain rate, $\Delta H$ and $V$ are the activation energy and activated volume, $T$ is the absolute temperature and $R$ is a physical constant. The formula shows that there is a linear relationship between $\sigma_{\mathrm{s}} / T$ and $\ln \dot{\varepsilon}_{\mathrm{s}}$.

Based on the concrete flowing mechanism, a model of polymer chain rotation conformation was proposed. ${ }^{9,10}$ It suggests the yield flow of molecule is caused by the rotation change of the polymer chain from the lower energy state of anti-form to the higher energy state of cis-form. When the proportion of the cismolecule is high enough, the macro-yield occurs.

Argon advanced another model of molecule flowing. ${ }^{11,12} \mathrm{He}$ suggested that the resistance of retarding the formation of double twisted chain is the elastic interaction between the chains. The yield process needs not only the formation of the double twisted chain but also the coordination of the neighbor chains. From this model, the relation between the yield shearing stress $\tau_{\mathrm{s}}$ and stain rate $\dot{\gamma}_{\mathrm{s}}$ can be obtained:

\footnotetext{
† To whom all correspondence should be addressed.
} 


$$
\tau_{\mathrm{s}}=\frac{0.102 G}{1-v}+\frac{16 \times 0.102 K T}{3 \pi \omega^{2} a^{3}} \ln \frac{\dot{\gamma}_{\mathrm{s}}}{\dot{\gamma}_{0}}
$$

where, $G$ is the shear modulus, $v$ is the Poisson's ratio, $a$ is the radius of the molecule chain, $\omega$ is rotation angle of the segment of the chain and $K$ is a physical constant.

Bowden analysed the yield sliding process of the non-crystalline polymers on the analogy of the expanding dislocation loops. ${ }^{13}$ The yield will occur when the radius of the loop reaches a critical value and the formation energy of the loop decreases with increase of the stress.

The free volume theory suggested that material may expand under stress field, which enhances the possibility of the chain segment's movement and results in yield. Some other theories indicated that many yield processes are caused by the micro-voids and micro-crack when the material expands. ${ }^{14-16}$ Many experiments show that in the process of yield, there are simultaneously molecule chain fracture, void formation, molecule orientation and molecule chain sliding. The more brittle a material is, the larger is the effect of the cracks and voids, and the tougher the material is, the larger is the action of the molecule orientation and sliding.

The present authors suggest that the yield mechanism of polymers does not depend only on the micro-structure revolution, but also on the dynamic process of the deformation region in the specimen. Thus, a yield model based on the multiplication of deformed region will be proposed and the corresponding elastic-plastic constitutive equation will be deduced.

\section{PHYSICAL MECHANISM OF DEFORMED REGION MULTIPLICATION}

Deformed region multiplication means that the deformation in a small region of the specimen can cause the deformation of its neighbor regions, which will make the farther regions deformed. In this conception, the deformation can expand to the whole specimen.

At the beginning of the loading, the polymers is in elastic stage. Then, due to the unavoidable geometric and structural non-uniformities, the plastic deformation occurs only in some stress concentration regions. The geometric nonuniformity is created during the preparation of the specimen, while the defects distributed at random will cause the structural non-uniformity. With the increase of stress, the plastic deformation in these regions causes new stress concentration, which will make of the autocatalytic effect of plastic deformation. In the molecule scale, the material is also inhomogeneous. The weak regions may contain a large amount of chain ends, untwisted section between the chains, and the sections chains vertical to the stress direction. Especially, when the chain is fractured, this position will become a new weak region. ${ }^{17}$ Under loading, these regions will be damaged first and formed micro cracks or voids, which makes their neighbor regions weakened. This mechanism of weak region multiplication is common in brittle materials.

The thermal effect in the deformation process will now be considered. When the strain rate is over $5 \times 10^{-3} \mathrm{~s}^{-1}$, the temperature increase can not be neglected. ${ }^{6}$ The heat may come from the work of the external forces, the decrease of entropy due to the molecule chain orientation and the internal energy release. The infro-red photography shows that the temperature in the shoulder region of the specimen is the highest. It means that there is a great quantity of heat in front of the plastic deformed region, and the neighbor elastic regions can be softened and create more heat due to the following plastic deformation, so the farther elastic regions will be softened until the plastic deformation expands to the whole specimen.

Another possible effect is that the radicals caused by the fracture of the tensional molecule chain can also become the catalyst for the 
frature of the neighbor molecule chain. ${ }^{18}$ This kind of failure can be regarded as one of the micro-mechanisms of deformed region multiplication.

On the basis of above analysis, the authors propose that the plastic deformation of polymers begins in some stress concentration positions and expands gradually by a mode of plastic deformed region multiplication. The dynamic characteristics of this process may result in the yield and cold tension. Hearle has indicated that ${ }^{19}$ in the view-point of microscope, the yield may concentrate on the shoulder regions or other local regions. The decrease of the yield stress may be caused by the changes of micro-structure. That is to say, once the structure starts to fail, the deformation of neighbor structure elements may be easier, or because the plastic deformation causes the material to heat.

\section{DEDUCTION OF THE CONSTITUTIVE EQUATION}

The following assumptions were proposed.

1) Multiplication rate is defined as the increment per unit volume of deformed region in unit time. It is a parameter related with the type of material.

2) The multiplication space (specimen volume) is limited, the material is isotropic and the dimensions of the specimen in three directions are the same order.

3) The time for keeping the multiplication is so long that the adjacent region can fully deform.

4) The time lag of the multiplication process is almost zero. This is based on the hypothesis that the velocity of heat transmitting is fast enough.

5) The induction period of multiplication (from initial loading to the beginning of plastic deformation in some regions) is small.

6) Multiplication damping and driving force are all constants during the whole deformation process.
7) Due to the finish machining of the specimen, the plastic deformation only starts at one position with highest stress concentration.

On the basis of above assumptions, the multiplication rate $\mathrm{d} \omega / \mathrm{d} t$ of the volume of the plastic deformed region is a function of the volume $\omega$. It increases from zero and reaches its maximum at a certain time, then decreases to zero due to the limitation of the multiplication space. So, we assume

$$
\frac{\mathrm{d} \omega}{\mathrm{d} t}=b \omega\left(1-\frac{\omega}{W}\right)
$$

By intergation, we get:

$$
\omega=\frac{W}{1+\mathrm{e}^{a-b t}}
$$

When $W=\omega, \mathrm{d} \omega / \mathrm{d} t=0$, so $W$ is the whole volume of the specimen. $\omega_{0}=\left.\omega\right|_{t=0}=W /(1+$ $\left.\mathrm{e}^{a}\right)$ is the volume of deformed region at initial time, and

$$
a=\ln \left(\frac{1}{C}-1\right)
$$

is a parameter related with $\omega_{0}$. Here, $C=$ $\omega_{0} / W$ is the initial volume ratio.

From eq 3, we get:

$$
\frac{1}{\omega} \cdot \frac{\mathrm{d} \omega}{\mathrm{d} t}=b\left(1-\frac{\omega}{W}\right)=B
$$

Here, $B$ is the actual multiplication rate for the above conditions. It will decrease when $\omega$ increases, which shows the limitation of the multiplication space. If the space is infinite, $\left.B\right|_{\omega \rightarrow \infty}=b$. Therefore, $b$ is a multiplication rate regardless of the space limitation. We assume it is a material constant.

In a real case, the effect of external stress $\sigma$ must also be considered. Under uniaxial loading, the larger the stress, the easier the multiplication. From this we assume that $\sigma$ is proportional to $\omega$, or proportional to the multiplication rate. The calculation shows that the second assumption is more reasonable. 
Therefore,

$$
\frac{\mathrm{d} \omega}{\mathrm{d} t}=b(\alpha \sigma+\beta) \omega\left(1-\frac{\omega}{W}\right)
$$

and

$$
\omega=\frac{W}{1+\mathrm{e}^{a-b(\alpha \sigma+\beta) t}}
$$

Here $\alpha$ and $\beta$ are constants determined by experiments.

The displacement $H$ of the crosshead of the tension machine is the sum of the elastic deformation $P$ of the tension machine and the deformation $Q$ of the specimen:

$$
H=V t=P+Q=\frac{\sigma S}{K}+Q \quad\left(t \geqslant t_{0}\right)
$$

In which, $V$ is the velocity of the crosshead, $t$ is the time of deformation, $t_{0}$ is the induction period of multiplication, $K$ is the elastic constant of the tension machine and $S$ is the cross area of the specimen.

For simplification, we assume the contribution $\psi$ of unit volume of the deformed region to the macro-extension of the specimen is a material constant. Therefore,

$$
Q=\frac{\sigma l}{E}+\omega \psi
$$

where $E$ is the elastic modulus. $l$ is the length of the elastic region along the tension direction, it can be expressed as

$$
l=\frac{W-\omega}{S}
$$

From eq 8-11 it follows

$$
V t=\frac{\sigma S}{K}+\frac{W\left\{\psi+\frac{\sigma}{E S} \exp \{a-b(\alpha \sigma+\beta) t]\right\}}{1+\exp [a-b(\alpha \sigma+\beta) t]}
$$

It is easy to rewrite the above relation between $\sigma$ and $t$ into stress-strain relationship. In fact, under constant velocity of loading, the two kinds of relation curves have similar shape.

For the discussion on the $\sigma-t$ relation, the elastic deformations of the tension machine and the specimen are neglected, we can get from eq 12 the following formula:

$\sigma=\frac{1}{\alpha b t}\left(a-\beta b t+\ln \frac{V t}{W \psi-V t}\right) \quad\left(t \geqslant t_{0}\right)$

\section{DISCUSSION ON THE $\sigma-t$ CURVE}

From eq 13, we can obtain:

$$
\begin{gathered}
\frac{\mathrm{d} \sigma}{\mathrm{d} t}=\frac{1}{\alpha b t^{2}}\left[\ln \left(\frac{W \psi}{V t}-1\right)+\frac{W \psi}{W \psi-V t}-a\right] \\
\left(t \geqslant t_{0}\right)
\end{gathered}
$$

By selecting $\mathrm{d} \sigma / \mathrm{d} t=0$, we get:

$$
\mathrm{e}^{-x}=a-x-1
$$

where $x=\ln ((W \psi / V t)-1)$. If $y_{1}=\mathrm{e}^{-x}, y_{2}=$ $a-x-1$, the relation between $y_{1}$ and $y_{2}$ has three possibilities in Descartes coordinate:

1) When $a<2$, the two curves of $y_{1}$ and $y_{2}$ do not intersect (Figure 1a), so there is not solution for eq 15, which follows that $y_{1}>y_{2}$ and $\mathrm{d} \sigma / \mathrm{d} t>0$. From formula $5, C=\omega_{0} / W>$ $1 /\left(1+\mathrm{e}^{2}\right)=11.9 \%$, i.e., when the initial volume ratio is bigger than $11.9 \%$, there are no extremums (or upper and lower stresses) in the $\sigma-t$ curve.

From eq 14 and $\mathrm{d}^{2} \sigma / \mathrm{d} t^{2}=0$, an equation of variable $t$ can be got:

$$
\begin{aligned}
\frac{W \psi(2 V t-W \psi)}{(W \psi-V t)^{2}}= & 2\left[\ln \left(\frac{W \psi}{V t}-1\right)\right. \\
& \left.+\frac{W \psi}{W \psi-V t}-a\right] \quad\left(t \geqslant t_{0}\right)
\end{aligned}
$$

According to calculation, there is also not solution for the above equation when $a<2$. That means, the curve has no the inflection point. Therefore, the eq 13 describes the yield mode shown in Figure 2a.

2) When $a=2$, there is an intersection point between $y_{1}$ and $y_{2}$ (Figure 1b). The real root 


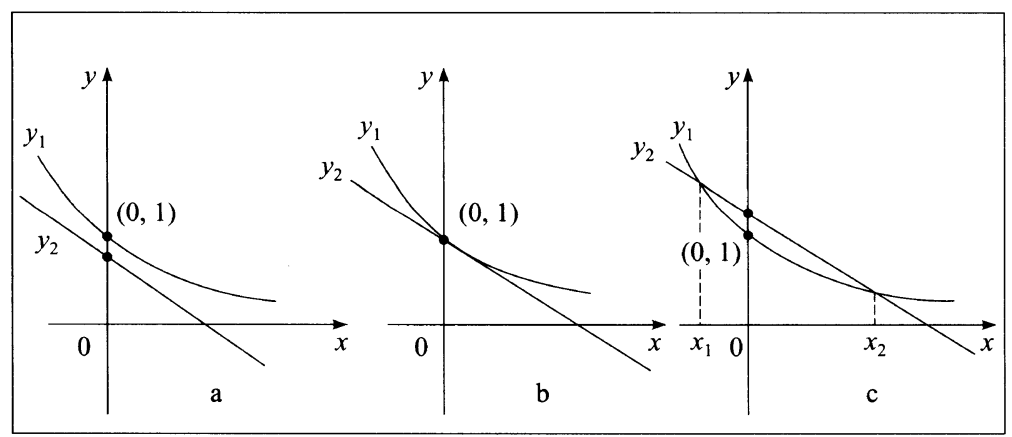

Figure 1. Three possible relations between the curves of $y_{1}$ and $y_{2}$.

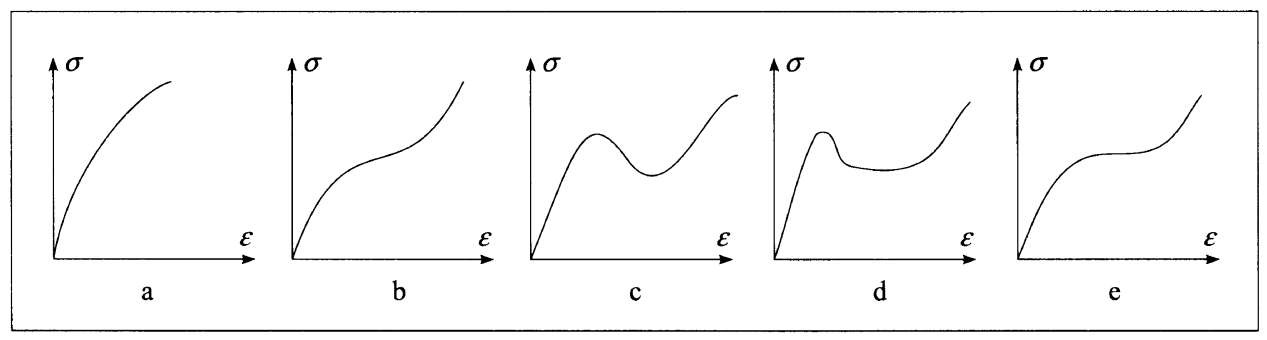

Figure 2. Several typical yield modes.

of eq 15 is $x=0$ when $t=W \psi / 2 V$. From eq 16 , this point is corresponding to the inflection point of the $\sigma-t$ curve, but not an extremum point. So, when the initial volume ratio is equal to the critical value $11.9 \%$, the eq 13 gives the yield mode shown in Figure $2 b$.

3) When $a>2$, there are two intersection points between $y_{1}$ and $y_{2}$ (Figure 1c). When $x_{2}<x, y_{1}>y_{2}$ and $\mathrm{d} \sigma / \mathrm{d} t>0$, which shows that before the tension time $t_{\mathrm{u}}$ corresponding to the second intersection point, $\sigma-t$ curve is upward. When $x_{1}<x<x_{2}, y_{1}<y_{2}$, so $\mathrm{d} \sigma / \mathrm{d} t<0$, the stress $\sigma$ will decrease. When $x<x_{1}$, the tension time is larger than $t_{\mathrm{L}}$ corresponding to the first intersection point, we get that $\mathrm{d} \sigma / \mathrm{d} t>0$ and the curve will go up again. Therefore, $t_{\mathrm{L}}$ and $t_{\mathrm{u}}$ are the time for lower and upper yield stresses respectively. The constitutive eq 13 describes the yield mode shown in Figure 2c.

According to the proceeding analysis, we can see that a well machined specimen with uniform micro-structure has a small region of plastic deformation at initial time, so $C$ is small or $a$ is large, there must exist obvious yield and plastic unstability. Otherwise, if the fabrication is crude, or the micro-structure is nonhomogeneous, there may be no upper and lower yield stresses, which is verified by the experiments.

On other hand, the cofiguration and size of the specimen can also affect the stress-strain curve. If the size in one direction is much smaller than in the other two directions, the specimen can be considered as a two dimensional plate. When the plate length and width are of the same order, it may form a deformed region in two dimensions. We assume:

$$
\frac{\mathrm{d} \omega}{\mathrm{d} t}=b \theta \omega^{1 / 2}\left(1-\frac{\omega}{W}\right)
$$

Where $\theta$ is the shape factor of the region. If the region extends circularly, $\theta=2 \sqrt{\pi}$. If it is a rectangle with the ratio $k$ between its length and width, $\theta=2\left(k^{1 / 2}+k^{-(1 / 2)}\right) . \theta$ is assumed as a constant during the deformation process. From eq 17, we get: 


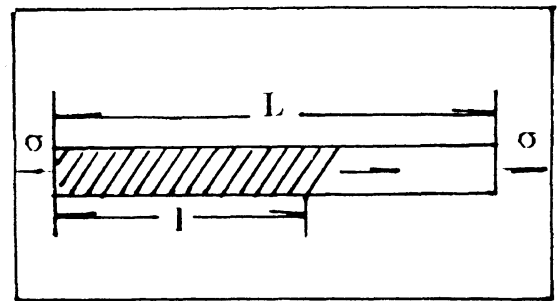

Figure 3. The extension of deformation band.

$$
\omega=W\left(\frac{C^{\prime} \mathrm{e}^{C^{\prime \prime} t}-1}{C^{\prime} \mathrm{e}^{C^{\prime \prime} t}+1}\right)^{2}
$$

where $C^{\prime}=\frac{1+C^{1 / 2}}{1-C^{1 / 2}}, C=\frac{\omega_{0}}{W}, C^{\prime \prime}=b \theta$. Assuming $b=b_{0}(\alpha \sigma+\beta)$ and neglecting the elastic deformation, we obtain:

$$
\sigma=-\frac{\beta \lambda t+\ln C^{\prime}}{\alpha \lambda t}+\ln D
$$

where $\lambda=b_{0} \theta, D=\frac{1+n^{1 / 2}}{1-n^{1 / 2}}, \quad n=\frac{V t}{\psi W}$.

If the size in tensile direction is much larger than those in other two directions, the initial three dimensional extension of deformed region will soon transform into one dimensional multiplication along the tensile direction. The deformation band will sweep the whole specimen shown in Figure 3. Let the length of the specimen be $L$, cross area be $S$, the length of deformed region be $l$. Because only the front of the deformed region can make its neighbor region deform, the multiplication velocity $\mathrm{d} \omega / \mathrm{d} t=S(\mathrm{~d} l / \mathrm{d} t)$ will not be proportional to the whole volume of the deformed region. It should be assumed that the larger the external stress, the faster the increasing of the length $l$ is. Consequently,

$$
\frac{\mathrm{d} \omega}{\mathrm{d} t}=S\left(\frac{\mathrm{d} l}{\mathrm{~d} t}\right)=b_{0}(\alpha \sigma+\beta)
$$

Regarding $\mathrm{d} l / \mathrm{d} t>0$ and $l=0$ as the initial conditions after the transformation from three dimensional multiplication to one dimensional extension. We get:

$$
\sigma=\frac{V S-\beta \psi b_{0}}{\alpha \psi b_{0}}
$$

It shows once one dimensional multiplication occurs, the stress will be constant. The corresponding stress-strain curve has a upper yield point and a yield platform, then, the stree will go up again after the deformation band extends to the whole specimen. So, most of specimens under uniaxial loading can have this kind of yield mode shown in Figure 2d.

If the fabrication is crude or the microstructure is non-uniform, the curve only has a platform without upper yield stress point, the mode is shown in Figure 2e.

It must be pointed out that the yield platform can be regarded as the equilibrium between the strain softening and the orientational intensification because $\psi$ has a limited value. But it can not be the result of the heat equilibrium in the shoulder area of the specimen.

For comparing with the experiments, we consider the strain rate:

$$
\dot{\varepsilon}=\frac{\mathrm{d} \varepsilon}{\mathrm{d} t}=\frac{1}{L} \cdot \frac{\mathrm{d} L}{\mathrm{~d} t}=\frac{V}{L}
$$

So

$$
V=L \dot{\varepsilon}
$$

From eq 13, we obtain

$\sigma_{\mathrm{s}}=\frac{a-\beta b t_{\mathrm{s}}}{\alpha b t_{\mathrm{s}}}-\frac{\ln \left(W \psi-L t_{\mathrm{s}} \dot{\varepsilon}\right)}{\alpha b t_{\mathrm{s}}}+\frac{\ln \left(L t_{\mathrm{s}} \dot{\varepsilon}\right)}{\alpha b t_{\mathrm{s}}}$

where $\sigma_{\mathrm{s}}$ is the yield stress, $t_{\mathrm{s}}$ is the occuring time of $\sigma_{\mathrm{s}}$. If $\dot{\varepsilon}$ is not too big and $t_{\mathrm{s}}$ is short enough, there is a linear relation between $\sigma_{\mathrm{s}}$ and $\ln \dot{\varepsilon}$, which is consistent with the microscopic yield models shown in formulas 1 and 2 , and is coincident with the experimental results $^{20}$ shown in Figure 4.

If $\dot{\varepsilon}$ is big enough, we will find that the relation of $\sigma_{\mathrm{s}}-\ln \dot{\varepsilon}$ is not completely linear. With the increasing of $\dot{\varepsilon}, \sigma_{\mathrm{s}}$ is larger than that of the value calculated from linear relation. Figure 5 is the experimental results of ref 21 . 


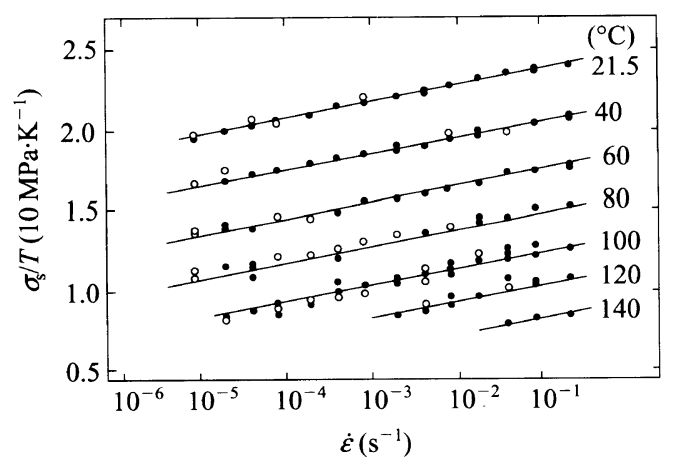

Figure 4. The relationship of $\sigma_{\mathrm{s}} / T-\dot{\varepsilon}$.

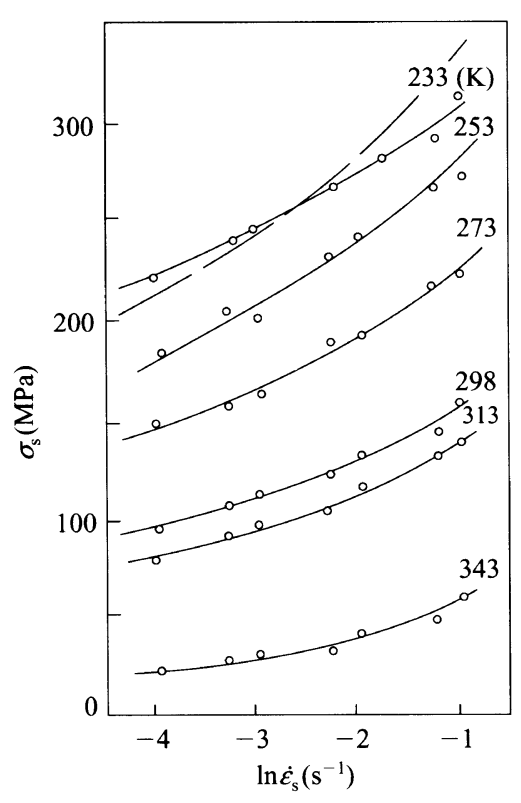

Figure 5. The relationship of $\sigma_{\mathrm{s}}-\ln \dot{\varepsilon}$.

Figure 6 shows our experimental results, the material is Poly(vinyl chloride) (PVC), $\sigma_{\mathrm{u}}$ is the upper yield stress. We consider the multiplication rate as a linear function of temperature:

$$
b=i T+j
$$

in which $T$ is the absolute temperature in the tests, $i$ and $j$ are the material constants. From eq 24 and 25, we obtained some calculated results which are basically consistent with the experiment shown in Figure 6.

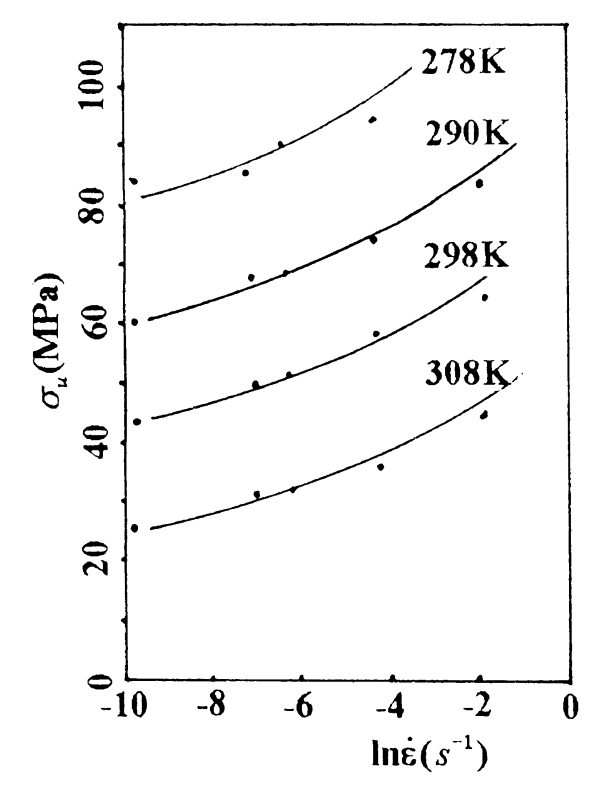

- experiment calculation

Figure 6. The relationship of $\sigma_{\mathrm{u}}-\ln \dot{\varepsilon}$ : $\bullet$, experiment; calculation.

\section{CONCLUSIONS}

1) The plastic deformation in polymers starts at local region. By the mode of multiplication, the deformed region will extend to the whole specimen. The front of the region forms the deformation band.

2) The phenomena of yield and cold flow in polymers are related with the multiplication process of the deformed region.

3) Under some conditions, a simple constitutive relationship can be deduced based on the multiplication process. Some quantitative descriptions on several typical yield modes can be got from different initial state, size and shape of the specimen.

4) Based on this model, the relation between yield stress and strain rate is consistent with the microscopic yield theories and the experimental results. 


\section{REFERENCES}

1. P. B. Bowden, in "The Physics of Glass Polymers," R. N. Haward, Ed., Applied Science Publishers, London, 1973.

2. W. Whitney and R. D. Andrews, J. Polym. Sci., C, 16, 2981 (1967)

3. N. Brown and I. M. Ward, J. Polym. Sci., A-2, 6: 607 (1968).

4. P. I. Vincent, Polymer, 1, 7 (1960).

5. S. W. Allison and I. M. Ward, Br. J. Appl. Phys., 18, 1151 (1967).

6. J. W. Maher, R. N. Haward, and J. N. Hay, J. Polym. Sci., Polym. Phys., 18, 2169 (1980).

7. R. N. Haward, Ed., "The Physics of Glassy Polymers," Applied Science Publishers, London, 1973.

8. A. J. Kinloch and R. J. Young, Fracture Behaviour of Polymers." Applied Science Publishers, London, 1983.

9. R. E. Robertson, J. Chem. Phys., 44, 3950 (1966).

10. R. A. Duckett, S. Rabinowitz, and I. M. Ward, $J$. Mater. Sci., 5, 909 (1970).
11. A. S. Argon, Phil May, 28, 839 (1973).

12. A. S. Argon and M. I. Bessonov, Polym. Eng. Sci., 17, 174 (1977).

13. P. B. Bowden and S. Raha, Phil May, 29, 144 (1974).

14. L. Nicholais and A. T. Di Benedetto, J. Appl. Polym. Sci., 15, 1585 (1971).

15. A. N. Gent, J. Polym. Sci., A-2, 10, 571 (1972).

16. A. N. Gent, J. Mater. Sci., 5, 925 (1970).

17. L. E. Nielsen, "Mechanical Properties of Polymers and Composites, Marcel Dekker Inc. New York, 1974.

18. S. N. Zhurkov, V. A. Zakrevskyi, V. E. Korsukov, and V. S. Kuksenko, J. Polym. Sci., A-2, 10, 1509 (1972).

19. J. W. S. Hearle, "Polymers and Its Properties" (in Chinese), Chendu Science and Technology University Publishing House, Chendu, 1988, p 211.

20. C. Bauwens-Crowet, J. A. Bauwens, and G. Hòmes, J. Polym. Sci., A-2, 7, 735 and 1745 (1969).

21. Chen Jiangying, Huang Xushen, Zhu Guorui, and Zhu Xixiong, "Journal of Ningbo University" (in Chinese), 3, 56 (1990). 